\title{
ECOG Performance Status 1
}

National Cancer Institute

\section{Source}

National Cancer Institute. ECOG Performance Status 1. NCI Thesaurus. Code C105723.

Restricted in physically strenuous activity but ambulatory and able to carry out work of a light or sedentary nature, e.g., light house work, office work. 